\title{
Effect of audible and visual reminders on adherence in glaucoma patients using a commercially available dosing aid
}

\author{
Lawrence $\mathrm{Y} \mathrm{Ho}^{\prime}$ \\ Larissa Camejo' \\ Malik Y Kahook ${ }^{2}$ \\ Robert Noecker' \\ 'UMPC Eye Center, University \\ of Pittsburgh School of Medicine, \\ Pittsburgh, PA I5213, USA; ${ }^{2}$ Rocky \\ Mountain Lions Institute, University \\ of Colorado, CO, USA
}

\begin{abstract}
We studied the effects of audible and visual alarms on adherence with a recommended dosing regimen in the management of glaucoma. Forty-two patients were begun on therapy with the Travatan ${ }^{\circledR}$ Dosing Aid (TDA) and randomly divided into two observation groups - one with visual and audible alarm functions turned on and the other with alarms off. Dosing information was analyzed for mean rates of adherence, missed days, and dosing at the wrong time. Twenty patients were randomized to the TDA alarm on group and 22 to the alarm off group. The rates of adherence were $87.9 \%$ and $79.7 \%(\mathrm{p}=0.02)$, rates of missed dosing were $7.6 \%$ and $14.4 \%$ $(\mathrm{p}=0.03)$, and rates of dosing at the incorrect times were $7.1 \%$ and $9.8 \%(\mathrm{p}=0.19)$, respectively for alarm on versus alarm off groups. In the alarm on group, the adherence rate was significantly higher and proportion of missed dosing was significantly lower. It is still yet to be determined whether there is a relationship between adherence and progression of glaucoma.
\end{abstract}

Keywords: adherence, glaucoma, Travatan ${ }^{\circledR}$ Dosing Aid, audible alarms, visual alarms

\section{Introduction}

Glaucoma is the second leading cause of blindness in the world according to the World Health Organization (Glaucoma Research Foundation). While there are multiple treatments for glaucoma, medical therapy is still the mainstay of therapy, especially in the early phases of the disease. Given the chronic and initially asymptomatic nature of this condition, adherence to prescribed treatment regimens is a significant problem for patients (Jackevicius et al 2002; Cramer et al 2003; Sewitch et al 2003; Osterberg and Blaschke 2005). Lack of consistency with dosing can lead to persistent elevation or fluctuations of intraocular pressure (IOP), which has been shown to be a risk factor for glaucomatous progression (Nouri-Mahdavi et al 2004). Therefore, improving patients' adherence to therapy is of the utmost importance.

There are many reasons for patients to be non-adherent to their treatment regimens (Patel and Spaeth 1995). Some of these include cost of medications, inability to dose the medications themselves, side effects, and failure to understand the role of medical therapy in the treatment of their disease. However, the most commonly cited reason for not dosing is patient forgetfulness (Patel and Spaeth 1995; Taylor et al 2002). An electronic medication-monitoring device, coupled with a reminder mechanism, allows physicians to indirectly measure adherence and may also decrease patient forgetfulness. However, the behavioral effects of these reminders, independent of other factors such as the intervention and measuring patient behavior, are yet to be determined.

Electronic monitoring is currently viewed as the most objective measure of a patient's adherence to a prescribed medical regimen (Farmer 1999). The date and time stamping mechanisms for the $\operatorname{Travatan}^{\circledR}$ Dosing Aid (TDA) (Alcon, Fort Worth, TX, USA) 
we used in this study has been shown to be both accurate and reproducible (Boden et al 2006; Cronin et al 2007). In this study, we examined how audible and visual alarms on a commercially available glaucoma medication dosing aid affected adherence to a monotherapy eyedrop dosing regimen.

\section{Methods}

Forty-two individuals newly diagnosed with glaucoma were studied. Eligibility criteria included any patient who presented to the glaucoma service between January 1, 2006 and January 1, 2007 who had never used topical glaucoma medications before and were willing to participate in the study. They were placed on monotherapy with travoprost (Travatan ${ }^{\circledR}$, Alcon, Fort Worth, TX). The basic design of the device has been previously described (Boden et al 2006). The TDA has an LCD screen that displays a flashing eye drop symbol on the front as a visible reminder when a patient is supposed to give themselves a dose of medication and it also emits an audible beep as an audible reminder. The patients were randomly assigned to begin once-daily dosing with this medication placed in the TDA with the alarm functions on or turned off.

Prior to use, the patient's name, demographic information, and prescribed dosing time were programmed into the device. In those cases in which the reminders were not used, the screen on the front of the TDA was covered with black tape and the audible alarm was turned off. In both situations, the device electronically monitored eye drop administration dates, times, and the number of drops administered (Figure 1).

Each patient was then given detailed instructions on the proper usage of the device. The dosing information was then downloaded from the TDA at the follow up visits 3-5 weeks later using the specialized cradle connected to a PC and a

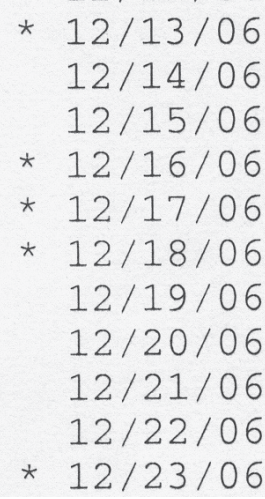

$06: 10$

$06: 19$

$09: 30$

$18: 17$

$06: 02$

$05: 49$

$06: 33$

$06: 55$

Figure I The dosing aid printout is divided into 3 columns that include date, time, and number of lever depressions. In this case the star to the left of the date denotes non-adherence to a programmed regimen. The absence of a star means the patient was adherent on that date. customized print out was obtained. Taking the medication outside the designated time frame was also noted as a nonadherence event and marked with a star on the printout (Figure 1). We analyzed the information for rates of adherence, missed dosing, dosing at the wrong time, double dosing, and range of time over which the drops were administered throughout the observation period.

In this study, adherence was defined as the number of days on which a drop was administered within 3 hours of the scheduled dose time regardless of whether or not one or more doses were administered that day. Non-adherence was defined as the number of days where no drop was administered at all or if no drops that day were administered within 3 hours of the scheduled dosing time. Dosing at the incorrect time was defined as administration of a drop outside the 3-hour window of the scheduled dose time. Comparisons were then made between the two groups using the nonparametric Kruskal-Wallis t-test and summary statistics.

\section{Results}

Of the 42 patients in our study, 23 were female and 19 were male. The age range was 42-91 years (mean, 69.1 \pm SD 12.5). Twenty patients were randomly assigned to the TDA alarm on group and 22 to the alarm off group. In the alarm on group, the observation period was 9-84 days and the mean duration of observation was $37.6 \pm 18.1$ days. In the alarm off group, the observation period was 6-63 days and the mean duration of observation was $35.4 \pm 16.2$ days $(p=0.28)$.

The adherence rates (Figure 2) were $87.9 \%$ and $79.7 \%$ $(p=0.02)$, rates of missed dosing (Figure 3 ) were $7.6 \%$ and $14.4 \%(\mathrm{p}=0.03)$, and rates of dosing at the incorrect times (Figure 4) were $7.1 \%$ and $9.8 \%(p=0.19)$, respectively for alarm on versus alarm off groups. The mean range of time between dose administration and scheduled dose time was $207 \pm 108$ minutes for the alarm on group and $424 \pm 405$ minutes for the alarm off group $(\mathrm{p}=0.08)$.

\section{Discussion}

In a recent evidence-based literature review of non-compliance with ocular hypotensive treatments, the proportion of patients who deviated from their prescribed medication regimen ranged from 5\% to $80 \%$ with several different measurements of compliance (Olthoff et al 2005). Compliance is classically defined as the extent to which patients' behaviors correspond with providers' recommendations (Schwartz 2005). We prefer the term adherence to therapy because it implies a more active patient participation and is more reflective of how medicine is practiced today. 


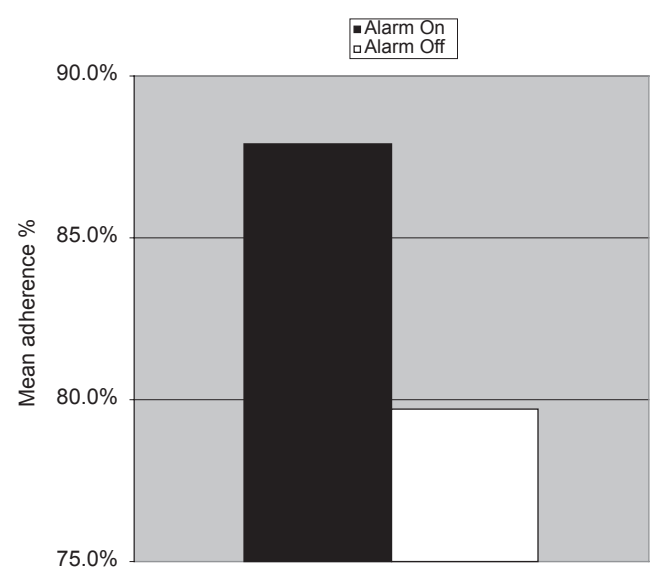

Figure 2 Mean adherence rate for those dosing travoprost with the alarm on $\mathbf{8 7 . 9 \%}$ compared with alarm off $79.7 \%$ was statistically significant $(p=0.02)$.

Methods for measuring adherence to glaucoma medications have included but are not limited to patient interviewing, questionnaires or self reporting, biomarkers, prescription refill rate, and electronic medication monitoring systems or combinations of the above (Kass et al 1986; Patel and Spaeth 1995; Tsai et al 2003; Nordstrom et al 2005; Boden et al 2006; Sleath et al 2006). The definition of adherence/compliance also varies across studies without any standardization.

In the present study, there were trends toward fewer days of dosing at incorrect times and a smaller average range of time between drop administration and scheduled dose time in the reminder group but these differences were not statistically significant. Some explanations for this lack of observed difference could be could be a relatively small sample size, short follow-up time, a biased population in that the patients had not used topical glaucoma therapy previously, and other factors such as new side effects may have come into play.

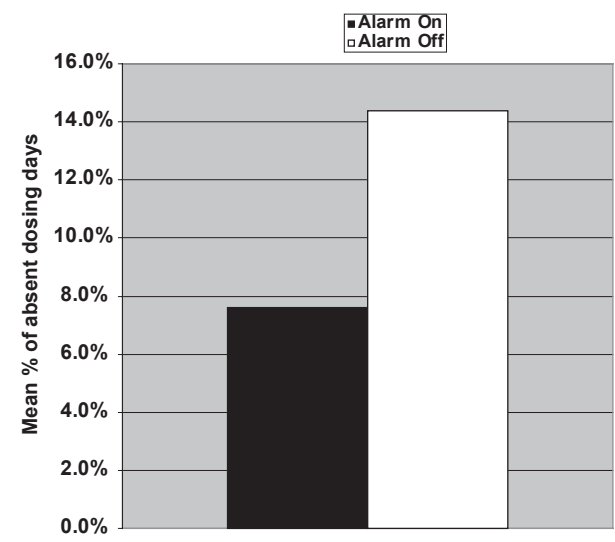

Figure 3 Mean rate of missed dosing for those on travoprost with the alarm on $7.6 \%$ compared with the alarm off $14.4 \%$ was statistically significant $(p=0.03)$.

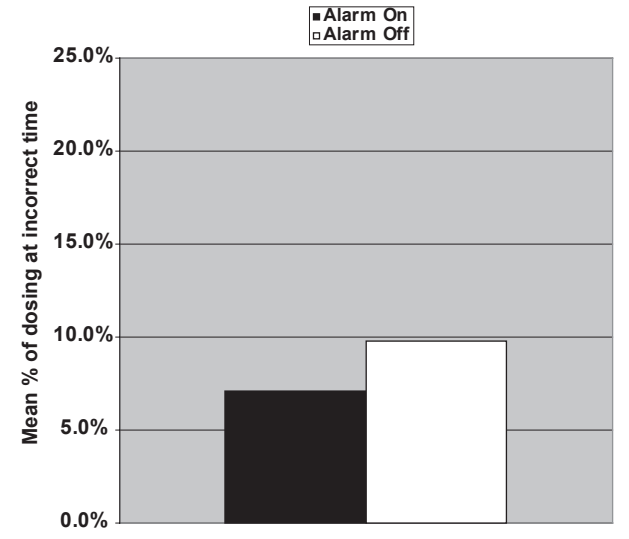

Figure 4 Mean rate for those dosing travoprost at the incorrect times with the alarm on $7.1 \%$ compared with the alarm off $9.8 \%$ was not statistically significant $(p=0.19)$.

Also, we did not randomize for situational/environmental factors (depression, race, marital status, children in household), medication regimens (the use of other systemic medications, medication side effects), patient related factors (general health, mentation, comprehension, vision, disability, health literacy), or provider related factors (duration of follow up), all of which have all been associated with lack of medication adherence (Tsai et al 2003; Tsai 2006). Another possible reason for the non-statistical significance is that when the patients are in a study they tend to be more compliant to their therapy (Lievens et al 2006).

The difference in the adherence rate and rate of missed doses was statistically significant between the two groups in our study. This suggests that the presence of the audible and visible alarms improved adherence and decreased the likelihood of missing a dose. Even if the patient was a sporadic user or did forget to dose at the appropriate time initially, the reminders may have rescued that day's dosing on some days.

The adherence rate for the reminder group was slightly higher than published overall rates of adherence but a recent study of adherence with the same monitoring device yielded similar rates of adherence (Kahook and Noecker 2007). Our rates may have been higher secondary to our sample size and our definition of adherence. We considered those patients who double dosed or dosed at the incorrect time to be adherent if they still administered one drop within 3 hours of scheduled time period. It is also impossible to assess whether or not the drop actually landed on the eye after a patient knowingly administered a drop. Another question not answered in our study was that the difference in reminder capability between audible and visual reminders. We did not separate the visual alarm from the audible alarm in order 
to see if one or the other was more effective on individual adherence rates. Nonetheless, the dosing device appeared to provide a reasonably accurate and objective method of recording adherence in these patients.

\section{Conclusion}

Monitoring of patient dosing behavior remains an elusive but attractive component of therapy for glaucoma. Being able to accurately determine the effect of patient adherence on therapy can be a powerful tool in determining which therapy works the best for a given individual. The effectiveness of reminders, as they currently exist, remains unclear but did appear to result in higher adherence rates and a diminished rate of missed dosing in this glaucoma population. It is still yet to be determined whether there is a relationship between adherence and progression of glaucoma. Larger prospective studies are needed to further elucidate the effect of visual and audible cues and their influence on adherence to glaucoma treatment regimens.

\section{Disclosures}

RJN and MYK have received speaking fees from Alcon. No funding was received for the research reported.

\section{References}

Boden C, Sit A, Weinreb RN. 2006. Accuracy of an electronic monitoring and reminder device for use with travoprost eye drops. J Glaucoma, 15:30-4.

Cramer J, Rosenheck R, Kirk G, et al. 2003. Medication compliance feedback and monitoring in a clinical trial: predictors and outcomes. Value Health, 6:566-73.

Cronin TH, Kahook MY, Lathrop KL, et al. Accuracy and performance of a commercially available dosing aid. Br J Ophthalmol, 91:497-9.

Farmer KC. 1999. Methods for measuring and monitoring medication regimen adherence in clinical trials and clinical practice. Clin Ther, 21:1074-90.
Glaucoma Research Foundation. Glaucoma facts and stats [online]. Accesssed Feb 6, 2007. URL: http://www.glaucoma.org/learn/facts.html.

Jackevicius CA, Mamdani M, Tu JV. 2002. Adherence with statin therapy in elderly patients with and without acute coronary syndromes. JAMA, 288:462-7.

Kahook MY, Noecker RJ. 2007. Evaluation of adherence to morning versus evening glaucoma medication dosing regimens. Clinical Ophthalmology, 1:79-83.

Kass MA, Meltzer DW, Gordon M, et al. 1986. Compliance with topical pilocarpine treatment. Am J Ophthalmol, 101:515-23.

Lievens CW, Gunvant P, Newman J, et al. 2006. Effect of Proview self-tonometry on pharmaceutical compliance. Clin Exp Optom, 89:381-5.

Nordstrom BL, Friedman DS, Mozaffari E et al. 2005. Persistence and adherence with topical glaucoma therapy. Am J Ophthalmol, 140:598-606.

Nouri-Mahdavi K, Hoffman D, Coleman AL, et al. 2004. Predictive factors for glaucomatous visual field progression in the Advanced Glaucoma Intervention Study. Ophthalmology, 111:1627-35.

Olthoff C, Schouten J, van de Borne B, et al. 2005. Noncompliance with ocular hypotensive treatment in patients with glaucoma or ocular hypertension. Ophthalmology, 112:953-61.

Osterberg L, Blaschke T. 2005. Adherence to medication. N Engl J Med, 353:487-97.

Patel SC, Spaeth GL. 1995. Compliance in patients prescribed eyedrops for glaucoma. Ophthalmic Surg, 26:233-6.

Patel SC, Spaeth Gl. 1995. Compliance in patients prescribed eyedrops for glaucoma. Ophthalmic Surg, 26:233-6.

Schwartz GF. 2005. Compliance and persistency in glaucoma follow-up treatment. Curr Opin Ophthalmol, 16:114-21.

Sewitch MJ, Abrahamowicz M, Barkun A, et al. 2003. Patient non-adherence to medication in inflammatory bowel disease. Am J Gastroenterol, 98:1535-44.

Sleath B, Robin AL, Covert D et al. 2006. Patient-reported behavior and problems in using glaucoma medications. Ophthalmology, 113:431-6.

Taylor SA, Galbraith SM, Mills RP. 2002. Causes of non-compliance with drug regimens in glaucoma patients: a qualitative study. $J$ Ocul Pharmacol Ther, 18:401-9.

Tsai JC. 2006. Medication adherence in glaucoma: approaches for optimizing patient compliance. Curr Opin Ophthalmol, 17:190-5.

Tsai JS, McClure CA, Ramos SE et al. 2003. Compliance barriers in glaucoma: A systematic classification. J Glaucoma, 12:393-8. 